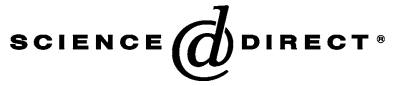

Cognitive Brain Research 23 (2005) 235-246
COGNITIVE

BRAIN

RESEARCH

www.elsevier.com/locate/cogbrainres

Research report

\title{
Imagining material versus geometric properties of objects: an fMRI study
}

\author{
Sharlene D. Newman ${ }^{\mathrm{a}, *}$, Roberta L. Klatzky ${ }^{\mathrm{b}}$, Susan J. Lederman ${ }^{\mathrm{c}}$, Marcel Adam Just ${ }^{\mathrm{b}}$ \\ ${ }^{a}$ Department of Psychology, Indiana University, 1101 E. 10th Street, Bloomington, IN 47405, USA \\ ${ }^{\mathrm{b} C a r n e g i e}$ Mellon University, Pittsburgh, PA, USA \\ ${ }^{\mathrm{c}}$ Queen's University, Kingston, ON Canada \\ Accepted 21 October 2004 \\ Available online 4 January 2005
}

\begin{abstract}
Two experiments are reported that used fMRI to compare the brain activation during the imagery of material and geometric object features. In the first experiment, participants were to mentally evaluate objects along either a material dimension (roughness, hardness and temperature; e.g., Which is harder, a potato or a mushroom?) or a geometric dimension (size and shape; e.g., Which is larger, a pumpkin or a cucumber?). In the second experiment, when given the name of an object and either a material (roughness and hardness) or geometric (size and shape) property participants rated the object on a scale from 1 to 4 . Both experiments were designed to examine the underlying neural substrate that supports the processing of material object properties with respect to geometric properties. Considering the relative amount of activation across the two types of object properties, we found that (1) the interrogation of geometric features differentially evokes visual imagery which involves the region in and around the intraparietal sulcus, (2) the interrogation of material features differentially evokes the processing of semantic object representations which involves the inferior extrastriate region, and (3) the lateral occipital cortex (LOC) responds to shape processing regardless of whether the feature being queried is a material or geometric feature.
\end{abstract}

(C) 2004 Elsevier B.V. All rights reserved.

Theme: Neural basis of behavior

Topic: Cognition

Keywords: fMRI; Haptic; Imagery; Object processing

\section{Introduction}

The mental representation of physical objects in human cognitive systems has been extensively studied from the dual perspectives of cognitive science and neuroscience. In previous research, however, the primary focus in object representation studies has been on visually perceptible attributes, even though objects have multiple attributes and can be recognized using other sensory modalities, particularly touch [21]. Here, two experiments are presented that use fMRI to explore memory-based processing not only for object properties that are salient to vision, such as the geometric properties of size and shape, but also properties

\footnotetext{
* Corresponding author.

E-mail address: sdnewman@indiana.edu (S.D. Newman).
}

that are salient to touch, such as the material properties of roughness and hardness.

A distinction between geometric and material properties has been documented in previous studies that contrasted visual and haptic perception. For example, Lederman and Klatzky [29] used a haptic search paradigm to demonstrate faster access to material properties (e.g., roughness, compliance, heat flow) than to geometric properties (e.g., edge and surface orientation). Additionally, a number of studies have shown relatively poor shape discrimination by touch $[3,28]$, whereas cutaneous and kinesthetic cues are essential for precisely discriminating material properties [42]. It has also been shown that when sorting objects by similarity, people place relative emphasis on geometric properties during sorting by visual similarity (even when touching the objects without vision) and on material properties during sorting by haptic similarity $[22,24,30]$. In a study directly 
related to the present work, Klatzky et al. [23] found that when asked to report about the properties of an object that could be seen and touched, people opted to use vision alone to determine geometric properties and make coarse judgments about material, but they additionally used touch to perceive material properties when precise discriminations were required.

Although behaviorally, the processing of geometric and material properties has been shown to rely differentially on the visual and haptic systems, recent neuroimaging studies have suggested considerable overlap in the underlying neural architecture responsible for visual and haptic processing, particularly as it relates to object geometry $[1,2,15,16,38]$. James et al. [15] found equivalent intramodal and cross-modal priming between visual and haptic presentations within the lateral occipital complex (LOC), which is located in the occipital-inferior temporal region. It has been suggested that the two modalities share a common underlying representation within LOC $[1,16]$, indicating a role for multi-sensory accessible memory representations. James et al. [16] argue that this common representation is related to object shape processing more generally.

Most previous neuroimaging studies of object processing, whether with real or remembered objects, using vision or touch, emphasized geometric object properties. An exception is by Servos, Lederman, Wilson, and Gati [41], who found activation in a common region of the post-central gyrus when objects were classified by shape, texture and hardness. Given that behavioral data show clear modality specialization in the processing of material and geometric object features, it appears that further emphasis in fMRI object-processing studies should be placed on differentiating the processing of geometry and material, in both perceptual and memory-based tasks.

The present study is particularly concerned with differentiating the processing of material and geometric properties when information about objects is retrieved from memory. This processing might or might not take the form of conscious imagery, be it visual or haptic. Indeed, the existence of a haptic form of imagery, independent of visual imagery, is not well documented. For example, in a previous behavioral study examining haptic judgments made from memory, Klatzky et al. [23] reported that subjects reported extensive use of visual imagery. Importantly, however, when making judgments about material features, they also frequently reported that their visual images contained a hand, often performing exploratory movements appropriate to the judgment being made (e.g., for a roughness judgment, rubbing the object's surface) [28]. Klatzky et al. conjectured that a form of haptic imagery might play a functional role in the judgments about material properties, despite its being manifested as a visual experience.

Several cortical regions of interest can be identified for the investigation of memory-based processing of objects, as follows.

\section{1. $L O C$}

As was mentioned above, LOC has been implicated in the multi-sensory processing of geometric object properties, particularly shape, using perception and memory tasks. These findings raise the issue of whether this region is more generally a multi-sensory object-processing area or whether it is specifically associated with shape processing. Object shape is critical to recognizing objects both visually and haptically, it may be that processing in LOC, an early point of convergence between modalities, pertains exclusively to shape. Therefore, LOC may be expected to be more involved when participants are asked about properties that can be determined by scrutinizing an object's shape regardless of whether it is a geometric or a material property.

\subsection{IPS}

Several studies have shown that the region in and around the intraparietal sulcus (IPS) is involved in visuo-spatial processing $[5,9,14,18,19,33]$, particularly as it relates to visual imagery $[14,19]$. It has been suggested that IPS is part of a top-down control mechanism during visual imagery that is primarily involved in maintaining the visual representation of an object retrieved from memory [14]. The intraparietal area has also been implicated in haptic/motor processing. For example, the anterior intraparietal area is associated with the control of hand movements during grasping, manipulation and the exploration of three-dimensional objects $[4,17]$. The posterior IPS, on the other hand, is thought to serve as a supra-modal integration center, especially for 3-D information relative to motor control, and is thought to be responsible for visual mental image generation $[4,17]$. Activation of IPS was found in tasks that required the determination of an object's length and curvature $[8,39]$.

\subsection{Semantic object representations}

When people are queried about the properties of familiar objects, pertaining either to material or geometry, they are likely to activate general knowledge about object properties. Both neuroimaging and lesion studies have implicated posterior ventral cortex, including inferior extrastriate (IES, which includes fusiform and lingual gyri), along with several other regions such as the temporal and prefrontal regions in semantic object processing $[11,13,14,32,43]$. In fact, IES was found to be involved when subjects match stimuli for semantic meaning as well as for structural properties suggesting that this occipital region is involved in a common semantic network [43]. A relative explanation for the involvement of IES in semantic object processing is that the region is involved in the processing of high-level perceptual descriptions of objects [13]. In the current study, it may be that when asked about properties that cannot be 
easily accessed via vision (or visual imagery), the object's perceptual description is searched to obtain the information. Therefore, one may predict greater involvement of IES for material compared to geometric queries.

\subsection{Somatosensory cortex}

Corresponding to the issue of whether visual cortex is involved in imagery of precise visual details, one can ask whether somatosensory cortex is activated when material properties are imagined. While several studies have found primary [6,31,38] and secondary [38] somatosensory cortex involved in tactile feature identification, the involvement of primary and secondary somatosensory cortex during memory-based material judgments has not been assessed.

\subsection{Premotor areas}

To the extent that people imagine actively exploring an object when making judgments about its properties from memory, one might expect premotor or even motor areas to become active. This might be particularly likely when material properties are judged, which has been found often to invoke an image of the moving hand [23].

\subsection{Visual cortex}

Memory-based processing of objects is often accompanied by the strong impression of visual imagery. It has been proposed that visual imagery corresponds to the activation of neural mechanisms normally involved in visual perception, although the precise locus has been a matter of some controversy. At least some studies have found activation in primary visual cortex, particularly when precise visual discriminations are required (see Kosslyn and Thompson, [25]).

Additionally, Sathian et al. have shown an important role for visual cortex, particularly the occipital/parietal portion, in tactile discrimination $[25,36,40,46]$. For example, disruption of occipital function with transcranial magnetic stimulation (TMS) was found to interfere with tactile discrimination of grating orientation [25]. These results have been taken to indicate a contribution of visual imagery to tactile discrimination. Visually based processing might possibly play a similar role in other tactile tasks such as tactile object recognition, especially when shape processing is critical.

To summarize the review thus far, a fundamental distinction has been made between geometric and material object properties, which differentially tie the processing of those properties to the visual and haptic modalities. Several brain areas, particularly those within visual cortex, have been implicated in the perceptual and memorial (including imaginal) processing of both haptic and visual inputs. However, fMRI studies have generally failed to evaluate the contribution of material properties as distinct from geometric ones.

The purpose of the current fMRI study is to further investigate how the brain processes geometric and material properties of objects from memory. The experimental approach is derived directly from the previous behavioral studies of Klatzky et al. [23]. Two experiments are reported here. The first is a comparison task in which participants were asked to mentally compare two objects along either a material dimension (e.g., Which is harder, a potato or a mushroom?) or a geometric dimension (e.g., Which is larger, a pumpkin or a cucumber?). The second study is an object-rating task in which participants were asked to rate objects on a scale from 1 to 4 along either a material dimension (roughness or hardness) or a geometric dimension (size or shape). The goal of these fMRI studies is to further explore differences in processing geometric and material properties of imagined objects. Of particular interest was the involvement of object processing and visual imagery when the two types of judgments were made.

\section{Experiment 1: comparison task}

\subsection{Participants}

Eleven neurologically normal, right-handed participants (4 males; mean age $=21$ ) from the Carnegie Mellon University community completed the current task during a fMRI session. All of the participants were paid volunteers who gave informed consent that was approved by the University of Pittsburgh and Carnegie Mellon Institutional Review Boards.

\subsection{Experimental paradigm}

Participants made judgments about either haptic or visual properties of pairs of objects. Participants were not explicitly instructed to generate a mental image. In all cases, the stimuli were written words. There were two geometric dimensions of comparison (size and shape) and three material dimensions (roughness, hardness and temperature, i.e., apparent warmth). On all of the dimensions of comparison, the items required comparisons between two objects that were perceptually close or distant from each other, to vary the difficulty of the judgment. Each trial consisted of a question followed by two object names.

Examples are:

\begin{tabular}{lll}
\hline $\begin{array}{l}\text { Which is rougher? } \\
\text { pear }\end{array}$ & Which is bigger? \\
\hline
\end{tabular}

The stimuli were projected onto a transparent screen that was suspended from the upper surface of the scanner bore. 
Each trial was presented for $4 \mathrm{~s}$ with an 8 -s delay between trials to allow for the offset of the hemodynamic response. The experiment consisted of sixty trials presented in a random order (i.e., 12 trials for each of the five conditions). In addition, four 24-s visual fixation periods were interspersed among the trials to obtain a control baseline measure of brain activation with which to compare the experimental conditions.

\section{3. fMRI procedure}

The study was conducted on a GE 3.0-T scanner used in conjunction with a commercial birdcage, quadrature-drive radio-frequency whole-head coil. Sixteen oblique-axial images were selected to maximize the coverage of the entire cortex. The images were collected using a spiral interleaved acquisition sequence, with $\mathrm{TR}=1000 \mathrm{~ms}$, TE $=$ $18 \mathrm{~ms}$, flip angle $=70^{\circ}$, and a $128 \times 64$ acquisition matrix with a voxel size of $3.135 \times 3.125 \times 3.125 \mathrm{~mm}$ with a 1 $\mathrm{mm}$ gap. The means of the images corresponding to each of the functional slices were registered to a high-resolution, T1-weighted structural volume scan of each participant. This volume scan was constructed from 124 3D SPGR axial images that were collected with $\mathrm{TR}=25 \mathrm{~ms}, \mathrm{TE}=4 \mathrm{~ms}, 40^{\circ}$ flip-angle, and a $24 \times 18 \mathrm{~cm} \mathrm{FOV}$, resulting in $0.9375 \times$ $0.9375 \mathrm{~mm} \times 1.5 \mathrm{~mm}$ voxels.

\section{4. fMRI data analysis}

Image preprocessing (including baseline correction, deghosting, mean correction, motion correction, and trend correction) was performed using FIASCO $[10,26]$; further description and tools are available at www.stat.cmu.edu/ $\sim$ fiasco/]. The mean of the maximum head motion per participant did not exceed 0.4 voxels. To ensure that the fMRI-measured activation was due to changes in cortical micro-vascular activity rather than changes in the bloodflow rate of larger vessels, any voxel that showed an excessively large percentage change in signal intensity (greater than 6.2\%) was excluded from the analyses.

To compare the activation across the experimental conditions in various regions, anatomical Regions of Interest (ROIs) were defined individually for each participant (this method has been reported previously, see Refs. $[5,18,19])$. This method provides more accurate anatomical localization than does morphing all participants' brains into a common space [34]. The ROIs were defined using the parcellation scheme of Rademacher et al. [7,37]. This method uses limiting sulci and coronal planes (defined by anatomical landmarks) to segment cortical regions. For each participant, a mean of the functional images was coregistered to the structural volume scan, in parallel alignment with the anterior commissure-posterior commissure (AC-PC) line. The limiting sulci and other anatomical landmarks were then located by viewing the structural images simultaneously in the three orthogonal planes, and the ROIs were defined by manually tracing the regions onto the axial image of each functional slice. The interrater reliability of this ROI-defining procedure between two trained staff members was previously evaluated for four ROIs in two participants in another study. The reliability measure was obtained by dividing the size of the set of voxels that overlapped between the two raters by the mean of their two set sizes. The resulting eight reliability measures were in the $78-91 \%$ range, with a mean of $84 \%$, as high as the reliability reported by the developers of the parcellation scheme (see Ref. [18] for further details).

The principal regions of interest included: (1) the posterior portion of the inferior temporal region defined by the parcellation scheme, which closely approximates LOC $[1,2,38]$, and will be called LOC for clarity; (2) the inferior extrastriate, which includes the fusiform and lingual gyri; (3) the intraparietal sulcal region; (4) premotor cortex, and (5) somatosensory cortex. Because the 16 functional slices collected covered most of the cerebral cortex, additional ROIs were drawn, and their data are reported for completeness.

The current study uses a common baseline approach. fMRI-measured activation was quantified for each experimental condition by first constructing a t-map comparing each voxel's intensity value in each experimental condition to the value in the baseline condition. Voxels with activation values exceeding baseline (determined by $t$ tests with $t>4$ ) were tabulated, providing the mean number of activated voxels (VC) within each ROI for each condition, a volumetric measure of activation. A second measure used was the mean percent increase (DSI) in the intensity of the activation relative to the baseline condition for those voxels included in the first measure. A third measure used was a combination of the first two. It is the sum of the changes in signal intensity (SSI) computed by adding the DSI for each voxel activated in a particular condition (see Ref. [45] for details). The activation measures across conditions are then submitted to an ANOVA.

The original behavioral study [23] found that imagery with haptic content (e.g., sight of the hand) was reported primarily in the difficult condition. The failure to report imagery during the easy condition does not necessarily indicate that no images were activated, only that the images were more salient during the difficult condition. In fact, the present activation patterns show no reason to separate difficult and easy items. A preliminary analysis showed high correlations between difficult and easy item sets with respect to the pattern of activation across regions for all five stimulus dimensions tested (average difficult/easy correlation $=0.87$ ). For that reason, as well as to increase statistical power in these analyses, difficult and easy items were combined for computing activation maps. Also, in the current comparison experiment, participants were not asked for image reports, therefore, we were unable to determine their conscious imagery. 


\section{Results}

\subsection{Behavioral measures}

The results support the assumption that the material and geometric tasks are quite comparable in informationprocessing demands. Although there was a trend for the reaction times to be longer to make the geometric comparisons than the material comparisons, this did not reach significance [material: $M=2359.8 \mathrm{~ms}$; geometric: $M=2441.5 \mathrm{~ms} ; F(1,10)=4.62, P<0.06]$. The accuracy of the responses in each condition was measured as the percentage agreement with norms for the responses in a previous study [23]. These agreement rates were not significantly different between property types [material: $M=80 \%$; geometric: $M=77 \% ; F(1,10)=1.94, P>0.1]$.

\section{2. fMRI results}

Although both material and geometric conditions activated a similar cortical network, several regions within that network revealed different levels of involvement as a function of condition. One example is IES, a cortical region associated with semantic object representations, which revealed greater activation levels when making material than geometric judgments, as predicted. Conversely, IPS, a region associated with visual imagery revealed greater activation for the geometric than material conditions (see Fig. 1). Other regions that showed differential activation included somatosensory, premotor, lexical/semantic, and executive processing regions. While several regions revealed a differential response to material and geometric judgments, the LOC showed similar levels of activation, as predicted by the multi-sensory hypothesis suggested by James et al. [16] and Amedi et al. [1,2].

\subsubsection{Inferior extrastriate and lateral occipital cortex (object processing)}

The IES and the LOC regions have been implicated in object processing, with IES being associated with semantic representations and LOC implicated in multi-sensory shape processing.

The activation within the IES was found to be significantly modulated by property type, with the material condition eliciting more activation than the geometric condition [left: $F(1,9)=9.95, P<0.05$; right: $F(1,9)=$ $3.13, P=0.11]$. Also, the activation within this region was found to be relatively bilateral, $[F(1,9)=1.96, P=0.2]$, as shown in Table 1.

Consistent with the multi-sensory role reported in previous studies $[1,2,15]$, the activation within LOC failed to show a significant effect of property type [left: $F(1,9)=$ 2.92, $P=0.12$; right: $F<1]$. The activation was found to be left lateralized $[F(1,9)=8.59, P<0.05]$. Also, the centroid of activation within the region is very similar to that found in Amedi et al. [1], $x=45 \pm 5, y=-62 \pm 6, z=-9 \pm 3$.

\subsubsection{Intraparietal sulcus (visual imagery)}

The IPS region was the only region that revealed significantly more activation for the geometric conditions

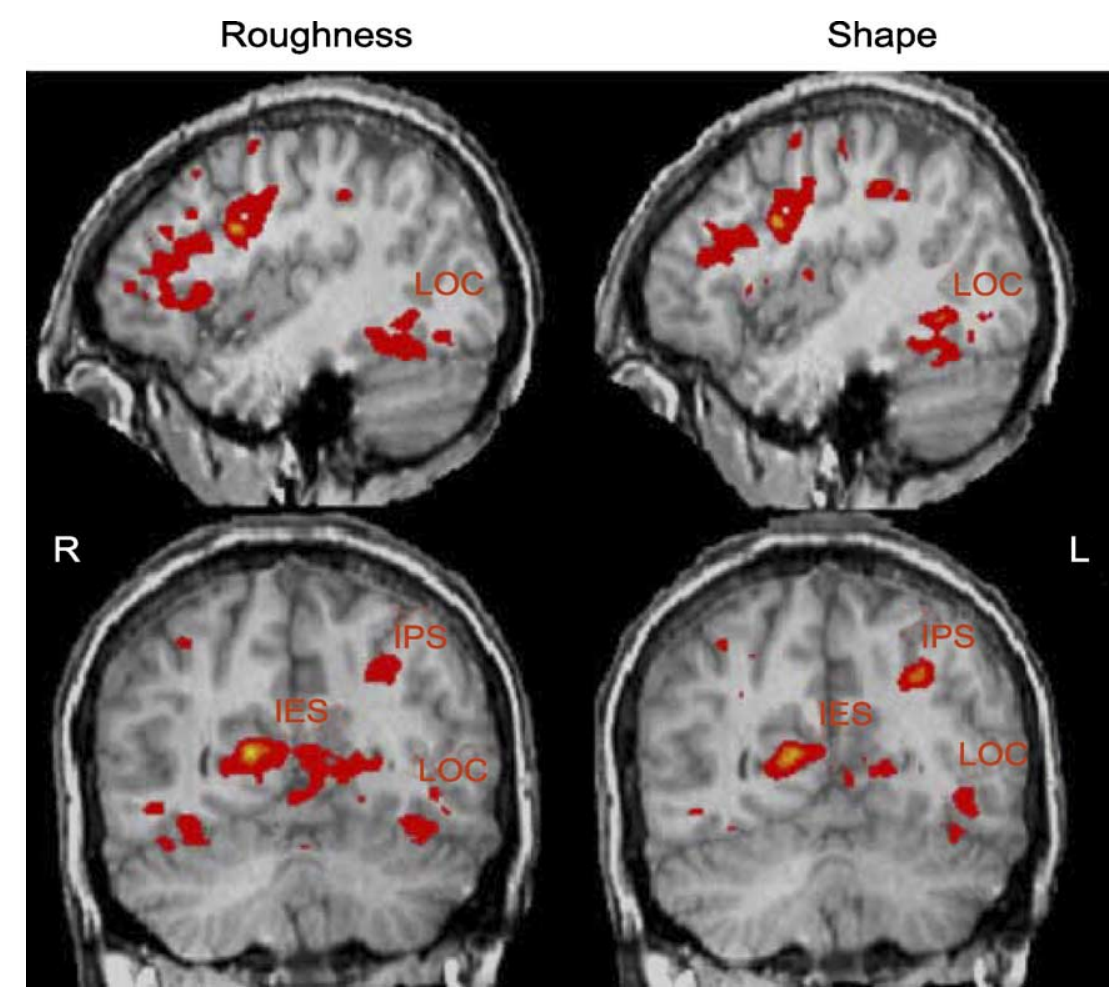

Fig. 1. Comparison study activation results. 
Table 1

Comparison experiment madality effects (Sum percent signal intensity)

\begin{tabular}{|c|c|c|c|c|c|c|c|c|c|c|}
\hline & \multicolumn{3}{|l|}{ Visual } & \multicolumn{4}{|l|}{ Haptic } & \multicolumn{3}{|c|}{ Talairach coordinates } \\
\hline & Shape & Size & $\begin{array}{l}\text { Average } \\
\text { visual }\end{array}$ & Roughness & Hardness & Temperature & $\begin{array}{l}\text { Average } \\
\text { haptic }\end{array}$ & $x$ & $y$ & $z$ \\
\hline \multicolumn{11}{|l|}{ Frontal lobe } \\
\hline Left dorsolateral prefrontal cortex & 8.7 & 15.9 & 12.3 & 31.8 & 18.7 & 15.5 & 22.0 & -32 & 31 & 30 \\
\hline Right dorsolateral prefrontal cortex & 6.4 & 11.9 & 9.2 & 21.3 & 8.1 & 8.2 & 12.5 & 31 & 35 & 31 \\
\hline Left frontal eyefields & 11.9 & 13.1 & 12.5 & 14.9 & 12.6 & 9.8 & 12.4 & -44 & 2 & 41 \\
\hline Right frontal eyefields & 1.6 & 2.3 & 1.9 & 2.8 & 0.8 & 1.4 & 1.6 & 39 & 7 & 41 \\
\hline Left pars opercularis & 14.9 & 9.8 & 12.4 & 19.3 & 16.9 & 12.4 & 16.2 & -41 & 11 & 21 \\
\hline Right pars opercularis & 5.1 & 4.0 & 4.5 & 9.5 & 4.7 & 5.0 & 6.4 & 39 & 12 & 16 \\
\hline Left pars triangularis & 15.5 & 9.2 & 12.3 & 27.3 & 16.6 & 16.4 & 20.1 & -41 & 25 & 16 \\
\hline Right pars triangularis & 2.1 & 1.3 & 1.7 & 8.0 & 2.6 & 1.5 & 4.0 & 39 & 23 & 11 \\
\hline Left premotor cortex & 9.7 & 6.5 & 8.1 & 11.4 & 8.3 & 4.5 & 8.1 & -44 & -3 & 35 \\
\hline Right premotor cortex & 1.7 & 2.9 & 2.3 & 7.2 & 4.4 & 1.3 & 4.3 & 41 & -8 & 44 \\
\hline Medial superior medial paracingulate & 17.0 & 13.9 & 15.4 & 26.2 & 14.8 & 14.8 & 18.6 & 0 & 21 & 45 \\
\hline Medial supplementary motor cortex & 3.0 & 1.0 & 2.0 & 5.2 & 0.8 & 2.4 & 2.8 & 0 & -7 & 59 \\
\hline \multicolumn{11}{|l|}{ Occipital/temporal lobe } \\
\hline Medial calcarine sulcus & 36.9 & 34.3 & 35.6 & 47.1 & 37.7 & 27.9 & 37.6 & 4 & -75 & 9 \\
\hline Left inferior extrastriate & 33.7 & 27.4 & 30.5 & 49.8 & 35.9 & 31.1 & 39.0 & -23 & -74 & -3 \\
\hline Right inferior extrastriate & 27.8 & 25.2 & 26.5 & 41.3 & 27.8 & 24.1 & 31.0 & 21 & -75 & 0 \\
\hline Medial occipital pole & 7.3 & 7.6 & 7.4 & 9.2 & 10.5 & 6.6 & 8.7 & 3 & -94 & 9 \\
\hline Left superior extrastriate & 6.1 & 3.8 & 4.9 & 9.1 & 4.6 & 3.4 & 5.7 & -18 & -80 & 22 \\
\hline Right superior extrastriate & 4.7 & 5.4 & 5.0 & 8.5 & 4.7 & 3.7 & 5.6 & 19 & -80 & 25 \\
\hline Left lateral occipital cortex & 16.7 & 14.4 & 15.5 & 26.5 & 15.6 & 12.9 & 18.3 & -43 & -56 & -4 \\
\hline Right lateral occipital cortex & 3.5 & 7.0 & 5.3 & 9.9 & 5.3 & 2.7 & 6.0 & 44 & -57 & -2 \\
\hline Left superior/middle temporal cortex & 4.7 & 4.2 & 4.5 & 10.9 & 5.4 & 5.5 & 7.3 & -51 & -16 & 5 \\
\hline Right superior/middle temporal cortex & 0.8 & 2.4 & 1.6 & 6.9 & 3.0 & 1.3 & 3.7 & 50 & -17 & 3 \\
\hline \multicolumn{11}{|l|}{ Parietal lobe } \\
\hline Left inferior parietal lobe & 11.9 & 16.4 & 14.2 & 18.0 & 10.8 & 11.0 & 13.3 & -48 & -50 & 26 \\
\hline Right inferior parietal lobe & 3.0 & 8.7 & 5.8 & 7.6 & 4.0 & 4.5 & 5.3 & 42 & -51 & 37 \\
\hline Left intraparietal sulcus & 20.1 & 21.6 & 20.9 & 24.9 & 13.6 & 14.1 & 17.5 & -28 & -60 & 42 \\
\hline Right intraparietal sulcus & 9.3 & 10.3 & 9.8 & 10.4 & 6.2 & 5.2 & 7.2 & 31 & -59 & 46 \\
\hline Left somatosensory cortex & 4.8 & 5.0 & 4.9 & 3.2 & 2.6 & 3.2 & 3.0 & -40 & -25 & 50 \\
\hline Right somatosensory cortex & 0.7 & 2.0 & 1.3 & 4.4 & 2.9 & 0.7 & 2.7 & 48 & -22 & 46 \\
\hline Left superior parietal lobe & 4.0 & 3.5 & 3.7 & 7.7 & 2.7 & 3.6 & 4.7 & -19 & -63 & 50 \\
\hline Right superior parietal lobe & 3.2 & 7.3 & 5.2 & 8.9 & 3.5 & 2.6 & 5.0 & 20 & -57 & 49 \\
\hline Left supramarginal gyrus & 2.6 & 2.2 & 2.4 & 2.8 & 2.7 & 1.8 & 2.4 & -50 & -29 & 40 \\
\hline Right supramarginal gyrus & 0.7 & 1.1 & 0.9 & 2.4 & 1.8 & 0.3 & 1.5 & 51 & -28 & 50 \\
\hline
\end{tabular}

than the material conditions [left: $F(1,9)=4.85, P=0.055$; right $F(1,9)=9.93, P<0.05]$. This is as predicted by the association of IPS with visual imagery, together with the assumption that visual imagery will particularly be evoked for geometric properties. It should be noted, however, that the material queries still generated significant activation relative to baseline in IPS, and the strongest activation level was actually found for a material property roughness. This is further consistent with the previous reports [23] that memory retrieval of material properties evoked visual imagery. The activation within IPS was also found to be significantly left lateralized, $F(1,10)=5.76, P<0.05($ Table 1$)$.

\subsubsection{Somatosensory and premotor areas}

The activation level within the somatosensory region was small in magnitude. However, both somatosensory and premotor regions revealed significant effects of property type. The activation within somatosensory cortex revealed an interaction between property type and hemisphere $[F(1,9)=$ 13.27, $P<0.01]$ with the left hemisphere showing more activation for the geometric condition $[F(1,9)=8.26, P<$ $0.05]$ and the right more activation for the material condition $[F(1,9)=8.16, P<0.05]$. The main effect of hemisphere was not significant $[F(1,9)=1.4, P>0.2]$.

The premotor cortex, on the other hand, revealed significant effects of property type, with the right hemisphere revealing more activation for the material condition than the geometric condition [left: $F<1$; right $F(1,9)=$ 5.15, $P<0.05]$. Also, the activation within the premotor cortex was significantly left lateralized $[F(1,10)=6.23, P<$ 0.05] (see Table 1).

\subsubsection{Inferior frontal cortex and posterior, superior temporal cortex (lexical/semantic processing)}

The activation within both the left posterior, superior temporal and the left frontal pars triangularis region was 
found to be significantly affected by property type, with the material conditions eliciting more activation than the geometric conditions [left temporal: $F(1,9)=19.39, P<0.005$; right temporal $F(1,9)=11.7, P<0.01$; left pars triangularis: $F(1,9)=7.15, P<0.05$; right pars triangularis: $F(1,9)=$ $3.51, P<0.1]$. The activation in both anterior and posterior regions was also found to be left lateralized [temporal: $F(1,10)=9.99, P<0.05$; pars triangularis: $F(1,10)=13.80$, $P<0.005$ ] (Table 1).

\subsubsection{Dorsolateral prefrontal cortex (executive processing)}

The activation within the dorsolateral prefrontal cortex revealed a significant effect of property type, with the material condition producing more activation than the geometric condition [left: $F(1,9)=9.07, P<0.05$;.15 right $F(1,9)=4.91, P=0.054]$. The activation was also found to be significantly left lateralized $[F(1,10)=6.75, P<0.05]$ (Table 1).

\subsubsection{Within dimension condition effects}

The roughness condition generally elicited more activation than either the hardness or the temperature condition. Significant differences $(P<0.05)$ were found in several ROIs including: the right DLPFC, left and right pars triangularis and temporal regions, left and right superior and inferior extrastriate, left and right premotor cortex, right IT, and left IPS. No such differences were observed for the two geometric dimensions.

\section{Discussion}

The primary goal of the current study was to examine differences in material versus geometric processing which we have hypothesized is related to IES and IPS activation, respectively. The findings here suggest that while memory retrieval of both material and geometric properties tends to activate the same cortical network, it relies differentially on the component nodes of that network. The greater activation in IES for the material than the geometric conditions is consistent with the idea that access to material features may rely more heavily on a semantic object representation. By contrast, the greater activation in the IPS region for the geometric conditions is consistent with that area's being associated with visual imagery and the assumption that geometric queries lead to greater visual imagery than material queries. That IPS activation was also found in the material condition is consistent with the Klatzky et al. [23] conclusion that both material and geometric conditions elicit visual imagery.

Statistically equivalent levels of activation for material and geometric judgments within LOC were found; the trend was actually toward greater activation for geometry than material. This result lends support for the hypothesis that the region is a multi-sensory region, in that it processes information related to both vision and touch $[1,2,15]$. It is important to note, however, that the evident involvement of LOC in memory-based judgments of material does not rule out its being specialized for processing shape, as it is possible that some material properties can be judged by shape information (e.g., roughness could be indicated by the visible sharpness of textured elements). We return to this point below.

There was also limited evidence to support the idea that primary sensory regions are involved in vivid recall of properties for which touch is specialized. While the extent of activation within the regions thought to be responsible for motor planning and somatosenory processing was small, the regions did reveal more activation during imagery of material properties compared to imagery of geometric properties in the right hemisphere, despite the absence of any haptic sensory input. The lateralization of this effect is intriguing, especially considering that the participants were all right-handed, but has no obvious explanation at present.

Differential activation was also observed in prefrontal cortex as well as in lexical/semantic processing regions. Because participants were asked to compare two objects and make a forced choice decision between the two, it may be that some of the differences observed are due to this decision process. To examine this issue, a second study was run in which the names of the same objects were again presented, but instead of comparing two objects along a given dimension, only one object was presented and participants were asked to rate the object with respect to either a material or geometric dimension. We expected to find diminished involvement of prefrontal cortical regions related to decision-making and left temporal regions related to lexical/semantic processing, while still observing geometric/material differences within the IES and IPS.

\section{Experiment 2: rating task}

Again, the purpose of the current fMRI study was to investigate how the brain processes geometric and material properties of objects from memory. Experiment 1 revealed that while memory retrieval of both material and geometric properties tends to activate the same cortical network, they rely differentially on the component nodes of that network, namely IES and IPS. Experiment 2 was designed to eliminate some of the decision-making processes in Experiment 1 in order to obtain a clearer picture of the cortical regions necessary for retrieving material and geometric properties from memory.

\subsection{Participants}

Seventeen neurologically normal, right-handed participants (9 males; mean age $=21.1$ ) from the Carnegie Mellon University community completed the current task during a 
fMRI session. All of the paid volunteers gave informed consent that was approved by the University of Pittsburgh and Carnegie Mellon Institutional Review Boards.

\subsection{Experimental paradigm}

The experiment involved participants making judgments about either material or geometric properties of objects. Participants were not explicitly instructed to generate a mental image. The stimuli were written words. There were two visual dimensions (size and shape) and two haptic dimensions (roughness and hardness). The objects were designed by pretest to be drawn approximately equally from the whole scale. Each trial consisted of a scale from 1 to 4 and an object name, such as:

\begin{tabular}{|c|c|c|c|}
\hline Soft & & & Hard \\
\hline 1 & 2 & 3 & 4 \\
\hline \multicolumn{4}{|c|}{ pillow } \\
\hline
\end{tabular}

The stimuli were projected onto a transparent screen that was suspended from the upper surface of the scanner bore. Each trial was presented for $4 \mathrm{~s}$ with an 8 -s delay between trials to allow for the offset of the hemodynamic response. The experiment consisted of 72 trials presented in a random order (i.e., 18 trials for each of the four conditions). In addition, four 24-s fixation periods were interspersed among the trials to obtain a control baseline measure of brain activation with which to compare the experimental conditions.

\section{3. fMRI procedure}

The data acquisition procedures were similar to that of the comparison study with the exception of the slice thickness, which was $5 \mathrm{~mm}$ here. The data analysis was similar to that of the comparison task reported above.

\section{Results and discussion}

\subsection{Behavioral measures}

The geometric items did not differ significantly from the material in reaction time (mean 2430 vs. $2462 \mathrm{~ms}$ ), $[F<1]$. Again, the two sets of items seem to have essentially equivalent processing demands.

\section{2. fMRI results}

The results of the rating experiment are very similar to those reported for the comparison experiment, showing property differences in several ROIs, including IES and IPS (see Table 2 and Fig. 2). As in the first experiment, the cortical region associated with object knowledge, IES, revealed more activation for the material condition while the region associated with mental imagery and spatial relations, IPS, revealed more activation for the geometric condition. LOC again showed a nonsignificant property difference, tending toward greater activation by material than geometry. As expected, when the comparison decision used in Experiment 1 was eliminated, condition effects within the lexical/semantic processing regions (i.e., left temporal cortex and left pars triangularis) and the prefrontal cortex were eliminated.

The drop-off in lexical/semantic and decision areas is not due to a decrease in reaction time because the response times were similar across experiments. It appears to reflect a change in the task components per se. Below is a description of the region-specific results.

\subsubsection{Inferior extrastriate and lateral occipital cortex (object processing)}

As in the comparison experiment, the activation within the IES was significantly modulated by property, with the material conditions eliciting more activation than the geometric conditions [left: $F(1,16)=6.67, P<0.05$; right: $F(1,16)=5.75, P<0.05]$. The activation within this region revealed a marginally significant effect of laterality, $[F(1,16)=3.86, P=0.067]$ (Table 2).

The LOC revealed a marginally significant difference between the material and geometric conditions (see Table 2) with the material condition eliciting greater activation than the geometric conditions [left: $F(1,16)=4.46, P=0.051$; right: $F(1,16)=3.68, P=0.073$ ]. The activation was also found to be left lateralized $[F(1,16)=6.77, P<0.05]$.

\subsubsection{Intraparietal cortex (visual imagery)}

As in the comparison experiment, the IPS was the only region that revealed significantly more activation for the geometric condition than the material condition [left: $F(1,16)=4.07, P=0.06$; right $F(1,16)=10.10, P<$ 0.01]. As before, material judgments generated significant activation in IPS; however, unlike Experiment 1, judgments of roughness did not produce stronger activation than geometric properties. The activation within IPS was also found to be significantly left lateralized, $F(1,16)=7.52, P<$ 0.05 (Table 2).

\subsubsection{Other regions}

As predicted, in contrast to Experiment 1, there were no significant effects of property type observed in lexical/ semantic processing regions [temporal cortex (left: $F(1,16)=$ $1.46, P=0.24$; right: $F<1)$, pars triangularis $(F$ 's $<1)]$ or prefrontal regions related to decision making [DLPFC (left: $F<1$; right: $F(1,16)=2.08, P=0.17)$ ] during this rating experiment. Experiment 2 also failed to show property effects in somatosensory cortex and pre-motor cortex [both $F$ 's $<1]$. However, like Experiment 1, the levels of activation of somatosensory and pre-motor areas were low, relative to IES and IPS. 
Table 2

Rating experiment maodality effects (Sum percent signal intensity)

\begin{tabular}{|c|c|c|c|c|c|c|c|c|c|}
\hline \multirow[t]{2}{*}{ Regions of interest } & \multicolumn{3}{|l|}{ Visual } & \multicolumn{3}{|l|}{ Haptic } & \multicolumn{3}{|c|}{ Talairach coordinates } \\
\hline & Shape & Size & Average visual & Roughness & Hardness & Average haptic & $x$ & $y$ & $z$ \\
\hline \multicolumn{10}{|l|}{ Frontal lobe } \\
\hline Left dorsolateral prefrontal cortex & 12.0 & 10.1 & 11.0 & 12.5 & 8.6 & 10.5 & -36 & 25 & 34 \\
\hline Right dorsolateral prefrontal cortex & 9.2 & 8.9 & 9.1 & 6.5 & 4.5 & 5.5 & 32 & 28 & 35 \\
\hline Left pars opercularis & 6.0 & 4.2 & 5.1 & 5.3 & 4.5 & 4.9 & -42 & 10 & 27 \\
\hline Right pars opercularis & 1.2 & 0.7 & 0.9 & 1.0 & 0.9 & 1.0 & 45 & 12 & 17 \\
\hline Left pars triangularis & 4.2 & 2.7 & 3.5 & 4.6 & 4.2 & 4.4 & -41 & 26 & 17 \\
\hline Right pars triangularis & 2.2 & 1.1 & 1.7 & 1.8 & 1.5 & 1.6 & 43 & 25 & 15 \\
\hline Left premotor cortex & 3.6 & 2.7 & 3.2 & 4.6 & 2.1 & 3.3 & -40 & -10 & 44 \\
\hline Right premotor cortex & 3.4 & 2.2 & 2.8 & 3.4 & 1.7 & 2.5 & 39 & -15 & 50 \\
\hline Medial superior medial paracingulate & 8.8 & 7.5 & 8.1 & 9.8 & 5.5 & 7.6 & 0 & 17 & 52 \\
\hline Medial supplementary motor area & 8.5 & 10.3 & 9.4 & 14.3 & 9.8 & 12.1 & 1 & -7 & 62 \\
\hline \multicolumn{10}{|l|}{ Occipital/temporal lobe } \\
\hline Medial calcarine sulcus & 42.0 & 23.9 & 32.9 & 35.7 & 27.1 & 31.4 & 4 & -71 & 9 \\
\hline Left inferior extrastriate & 19.3 & 12.1 & 15.7 & 25.8 & 15.0 & 20.4 & -23 & -68 & -6 \\
\hline Right inferior extrastriate & 12.6 & 7.4 & 10.0 & 17.2 & 10.5 & 13.9 & 25 & -67 & -6 \\
\hline Medial occipital pole & 13.3 & 8.8 & 11.0 & 13.2 & 9.9 & 11.5 & 0 & -90 & 6 \\
\hline Left superior extrastriate & 4.4 & 4.1 & 4.2 & 5.1 & 2.7 & 3.9 & -16 & -80 & 32 \\
\hline Right superior extrastriate & 2.7 & 2.5 & 2.6 & 3.1 & 2.3 & 2.7 & 20 & -77 & 33 \\
\hline Left lateral occipital cortex & 6.0 & 3.1 & 4.5 & 8.0 & 3.4 & 5.7 & -45 & -53 & -3 \\
\hline Right lateral occipital cortex & 2.1 & 1.7 & 1.9 & 3.4 & 2.2 & 2.8 & 49 & -50 & -4 \\
\hline Left superior/middle temporal cortex & 1.4 & 0.7 & 1.1 & 2.8 & 0.9 & 1.9 & -51 & -22 & 8 \\
\hline Right superior/middle temporal cortex & 1.2 & 0.8 & 1.0 & 1.6 & 0.4 & 1.0 & 50 & -16 & 8 \\
\hline \multicolumn{10}{|l|}{ Parietal lobe } \\
\hline Left inferior parietal lobe & 9.2 & 8.1 & 8.6 & 8.3 & 6.0 & 7.1 & -39 & -54 & 45 \\
\hline Right inferior parietal lobe & 4.2 & 3.7 & 4.0 & 3.0 & 2.2 & 2.6 & 46 & -47 & 42 \\
\hline Left intraparietal sulcus & 24.8 & 21.8 & 23.3 & 20.7 & 18.9 & 19.8 & -27 & -61 & 46 \\
\hline Right intraparietal sulcus & 16.7 & 16.7 & 16.7 & 12.7 & 10.4 & 11.6 & 33 & -59 & 45 \\
\hline Left somatosensory cortex & 7.8 & 7.1 & 7.4 & 10.0 & 5.0 & 7.5 & -41 & -27 & 51 \\
\hline Right somatosensory cortex & 8.4 & 6.5 & 7.5 & 10.2 & 5.7 & 7.9 & 40 & -28 & 53 \\
\hline Left superior parietal lobe & 11.6 & 11.9 & 11.8 & 13.3 & 9.4 & 11.3 & -15 & -63 & 53 \\
\hline Right superior parietal lobe & 10.6 & 13.9 & 12.3 & 15.5 & 8.9 & 12.2 & 22 & -58 & 52 \\
\hline Left supramarginal gyrus & 7.7 & 5.5 & 6.6 & 8.5 & 7.3 & 7.9 & -48 & -32 & 43 \\
\hline Right supramarginal gyrus & 3.9 & 3.6 & 3.8 & 4.6 & 3.2 & 3.9 & 50 & -27 & 43 \\
\hline
\end{tabular}

\subsubsection{Within-dimension condition effects}

As in the comparison experiment, several ROIs revealed significant differences $(P<0.05)$ between the activation related to roughness and hardness, with roughness showing greater activation. These regions included left and right DLPFC, left and right temporal cortex, left and right superior and inferior extrastriate, left IT, left IPS, left and right premotor cortex, left and right somatosensory cortex, and left and right superior and inferior parietal cortex. The geometric conditions (shape and size) revealed significant differences $(P<0.05)$ within the following ROIs: the calcarine sulcus, the occipital pole, left and right IES and left IT, with shape eliciting greater activation.

\section{General discussion}

To our knowledge, this is the first fMRI study contrasting memory-based judgments of material and geometric object properties, which are more strongly associated with haptic and visual perception, respectively. The results are exciting in that they may be the first to show that a visual processing region, the inferior extrastriate area (IES), is intimately involved in the processing of the material properties of objects as well as their geometric properties. The results also show that, although retrieval of material and geometric object properties elicit a similar pattern of cortical activation, they rely differentially on two different cortical regions. The material condition elicited significantly more activation within a region that may be related to the semantic representation of objects, the inferior extrastriate, than did the geometric condition. Conversely, the geometric condition elicited more activation within a region associated with visuo-spatial processing, the region in and around the intraparietal sulcus (IPS). The lateral occipital cortex (LOC), associated with multisensory processing in perception and memory, was similarly activated equally by material and geometric judgments. The discussion below addresses how the results speak to the manner in which object features may be stored and/or accessed. 


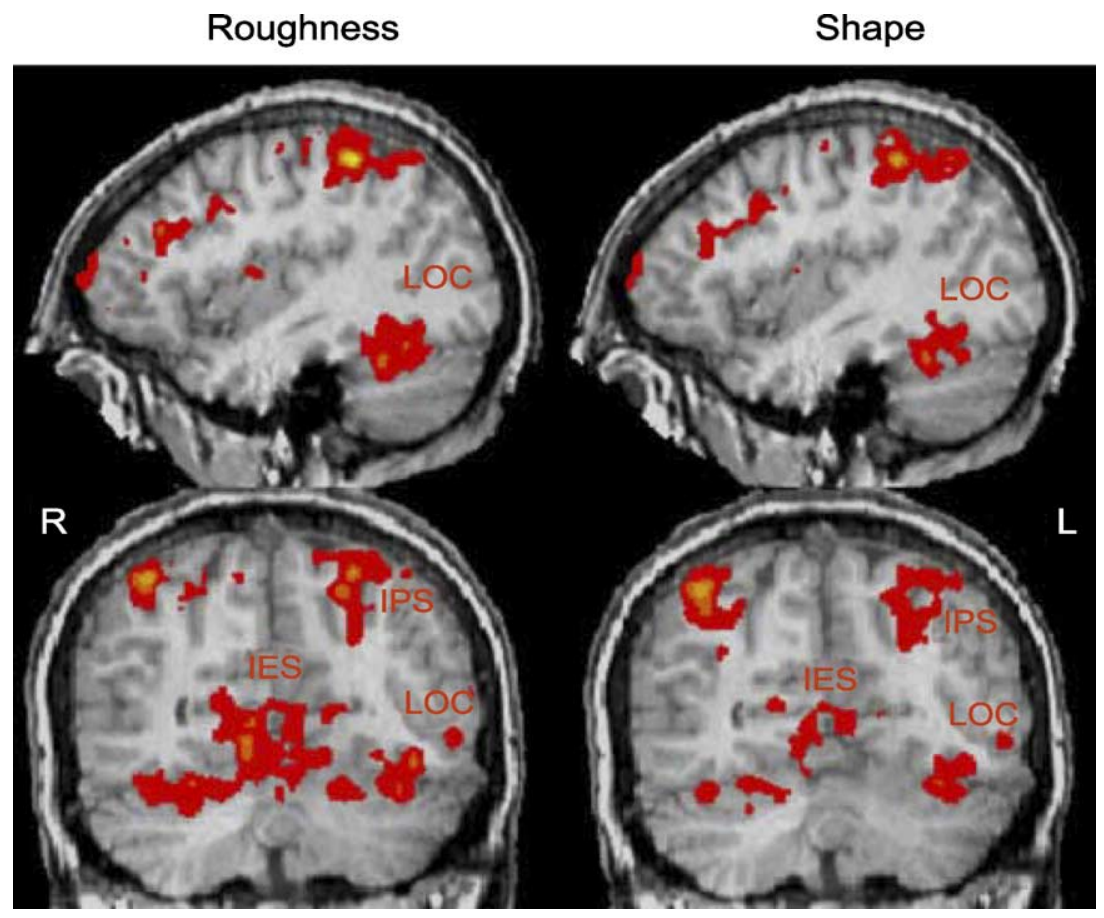

Fig. 2. Rating study activation results.

\subsection{Object processing}

All of the objects examined here were familiar and therefore had a representation stored in memory. One explanation for the increased involvement of IES when making material judgments is that IES is involved in the processing of semantic object representations and activation of the region was evoked by the retrieval and/or the evaluation of object attributes. This suggests that the greater involvement of IES in answering material queries is due to retrieving information regarding both material and geometric properties while the visual queries entail only retrieving the geometric information.

Additionally, the involvement of IES in material judgments might also reflect contributions of non-visual imagery, such as haptic or motor sensations. It is worth noting that reports of such images were found by Klatzky et al. [23] only when material properties were judged (e.g., participants imagined performing exploratory movements appropriate to the judgment being made). In any case, the data do support the idea that IES is involved in the processing of both material and geometric object features, at least for familiar objects.

\subsection{Visual imagery}

There has been some debate related to the dependence of haptic processing on visual processing, particularly visual imagery $[2,8,16,36,40,46]$. The current study found that the geometric conditions elicited greater involvement of the intraparietal sulcus (IPS), a region associated with higher level visuo-spatial processing, particularly with respect to visual imagery. The greater IPS activation for geometric than for material judgments suggests, then, a greater involvement of visual imagery in geometric than in material judgments.

Although IPS activation was greater on average for geometric judgments, substantial activation was also observed for material judgments, particularly roughness. Visual imagery could function in multiple ways in judgments about material properties. First, it may represent the layout of geometric features associated with material, such as the sharpness of elements in a rough texture or the rounded contours of a soft object (see Ref. [35]). A second role, which is suggested by the previous studies of Klatzky et al. [23], is that sight of the hand in a visual image may provide information about material. For example, the rate at which a hand is imagined to deform an object's contours may provide a cue to softness. A third possibility is that visual imagery is a subjective concomitant of non-visual forms of imagery that contribute to the judgment of object properties. Examples of non-visual imagery could include cutaneous, kinesthetic, and/or motor components. Although reports of imagined haptic or motor sensations were relatively infrequent in the Klatzky et al. [23] study, some subjects reported such feelings when material properties were being judged. The visual imagery in IPS could also portray movements of the hand, or it could arise in conjunction with non-visual forms of imagery. For example, Heller [12] showed that roughness perception is aided by the sight of the hand, despite having visual surface texture cues eliminated. 


\subsection{LOC: a multi-sensory region}

James et al. [15,16], as well as Amedi et al. [1,2], have argued that LOC is a multi-sensory processing region responsible for shape processing regardless of the sensory modality used, visual or haptic. The current study found that there was little effect of property type (only marginal significance in Experiment 2, with material producing greater activation than geometry). The finding that judgments of both material and geometry activate LOC is consistent with the idea that it is accessed by both the visual and haptic modalities. A substantial role for LOC in material judgments does not rule out the idea that the area is specialized for shape processing. This is because the two object properties that elicited the greatest amount of activation from the region are shape and roughness, both of which may be expected to rely heavily on shape processing (visible surface properties can lead to reliable and orderly judgments of roughness magnitude [27]). This finding extends the function of LOC proposed by James et al. [16]. James et al. $[15,16]$ did not vary the material properties of objects so he cannot say what would happen if material properties were also varied. Here, it has been shown that LOC becomes increasingly involved when processing shape information, regardless of whether the information is more readily accessed visually or haptically. Further investigation of the association between LOC and shape processing is needed.

\subsection{Differences within the material dimensions}

Generally, questions about roughness elicited more activation than either the hardness or temperature condition, in both IES and IPS. It should be noted in this regard that material properties are far from uniform. Within the perceptual system, material properties differ in the nature of the peripheral receptors that mediate the perceptual outcome, the pattern of exploratory movements used to seek the properties, and the nature and time-course of computations that are done on the basis of peripheral signals (see Ref. [20], for a review). Considering memorybased processing, including imagery, haptically accessible properties differ in the visual cues with which they are associated. Roughness can be signaled by the fine-grained micro-geometric surface features of an object, such as the bumps on an orange peel that differentiates it from a smooth apple. In contrast, temperature and hardness are not obviously apparent from an object's geometric features. No doubt these differences would affect the contributions of neural structures to memory retrieval of material features.

\subsection{Sensory activation during vivid remembering}

While there have been several studies showing activation of primary visual cortex during imagery, the current study found relatively little activation of somatosensory cortex during material judgments, although Experiment 1 did show a property type effect favoring the material condition in right somatosensory and premotor cortex. It should also be noted that there was no significant difference as a function of property within primary visual cortex (the calcarine sulcus). These findings are consistent with others which found that during vivid recall, it was primarily the secondary sensory regions that became reactivated, as opposed to primary sensory cortex [e.g., [44]]. The present results, on the whole, fail to show strong evidence that vivid recall of material information leads to activation of cortical areas that process haptic perceptual information at early stages.

\subsection{Summary and conclusions}

In summary, our results indicate that when properties of familiar objects are to be retrieved from memory several brain regions, most notably IPS, IES, and LOC, are activated. Considering the relative amount of activation across the two types of object properties, we find that (1) the interrogation of geometric features differentially evokes visual imagery which involves the IPS, (2) the interrogation of material features differentially evokes the processing of semantic object representations which involves the IES, and (3) LOC responds to shape processing regardless of whether the feature being queried is a material or geometric feature.

The findings reported here also suggest several questions on which to base future work. A critical issue is the type of information processed in IPS, IES, and LOC when judgments of material are made. How is material information represented; that is, is it always structurally mediated? For example, if asked to imagine softness would we imagine structural cues to softness such as rounded edges? Or would we experience the tactile or kinesthetic softness? This issue is particularly relevant to LOC and IPS, which have been characterized as shape-processing regions. Another issue is whether the involvement of these brain regions could be modulated by the familiarity of the objects and hence the availability of information in memory, as would be expected in particular from the semantic region IES. Still another question is whether material and geometric queries elicit different processing strategies, promote the retrieval of different types of information, or both. For example, do you think the data presented suggest that semantic processing is more salient for material relative to geometry? The current study constitutes a first step in investigating these and other issues related to the cognitive and neural representation of material properties.

\section{References}

[1] A. Amedi, R. Malach, T. Hendler, S. Peled, E. Zohary, Visuo-haptic object-related activation in the ventral visual pathway, Nat. Neurosci. 4 (2001) 324-330. 
[2] A. Amedi, G. Jacobson, T. Hendler, R. Malach, E. Zohary, Convergence of visual and tactile shape processing in the human lateral occipital complex, Cereb. Cortex 12 (11) (2002) 1202-1213.

[3] I. Biederman, Recognition-by-components: a theory of human image understanding, Psychol. Rev. 94 (1987) 115-148.

[4] F. Binofski, G. Buccino, S. Posse, R.J. Seitz, G. Rizzolatti, H. Freund, Fronto-parietal circuit for object manipulation in man: evidence from an fMRI study, Eur. J. Neurosci. 11 (1999) 3276-3286.

[5] P.A. Carpenter, M.A. Just, T.A. Keller, W.F. Eddy, K.R. Thulborn, Graded functional activation in the visuo-spatial system with the amount of task demand, J. Cogn. Neurosci. 11 (1999) 9-24.

[6] R.J. Caselli, Rediscovering tactile agnosia, Mayo Clin. Proc. 66 (1991) 129-142.

[7] V.S. Caviness Jr., J. Meyer, N. Makris, D.N. Kennedy, MRI-based topographic parcellation of human neocortex: an anatomically specified method with estimate of reliability, J. Cogn. Neurosci. 8 (1996) 566

[8] E. Deibert, M. Kraut, S. Kremen, J. Hart, Neural pathways in tactile object recognition, Neurology 52 (1999) 1413-1417.

[9] V.A. Diwadkar, P.A. Carpenter, M.A. Just, Collaborative activity between parietal and dorso-lateral prefrontal cortex in dynamic spatial working memory revealed by fMRI, NeuroImage 12 (2000) $85-99$.

[10] W. Eddy, M. Fitzgerald, C. Genovese, A. Mockus, D. Noll, Functional imaging analysis software-computational olio, In Proceedings in Computational Statistics, Physica-Verlag, Heidelberg, 1996, pp. 39-49.

[11] G. Gainotti, M.C. Silveri, A. Daniele, L. Giustolisi, Neuroanatomical correlates of category-specific impairments: a critical survey, Memory 3 (1995) 247-264.

[12] M.A. Heller, Visual and tactual texture perception: intersensory cooperation, Percept. Psychophys. 31 (1982) 339-344.

[13] G.W. Humphreys, Object recognition: the man who mistook his dog for a cat, Curr. Biol. 6 (1996) 821-824.

[14] A. Ishai, L.G. Ungerleider, J.V. Haxby, Distributed neural systems for the generation of visual images, Neuron 28 (2000) 979-990.

[15] T.W. James, K. Humphrey, J.S. Gati, P. Servos, R.S. Menon, M.A. Goodale, Haptic study of three-dimensional objects activates extrastriate visual areas, 10 (2002).

[16] T.W. James, K.H. James, G.K. Humphrey, M.A. Goodale, Do visual and tactile object representations share the same neural substrate? in: M.A. Heller, S. Ballesteros (Eds.), Touch and Blindness: Psychology and Neuroscience, Lawrence Erlbaum, Mahwah, NJ, 2004.

[17] L. Jäncke, A. Kleinschmidt, S. Mirzazade, N.J. Shah, H.-J. Freund, The role of the inferior parietal cortex in linking the tactile perception and manual construction of object shapes, Cereb. Cortex 11 (2001) $114-122$

[18] M.A. Just, P.A. Carpenter, M. Maguire, V. Diwadkar, S. McMains, Mental rotation of objects retrieved from memory: an fMRI study of spatial processing, J. Exp. Psychol. Gen. 130 (2001) 493-504.

[19] M.A. Just, S.D. Newman, T.A. Keller, A. McEleney, P.A. Carpenter, Imagery in sentence comprehension: an fMRI study, NeuroImage (2004).

[20] R.L. Klatzky, S.J. Lederman, Touch, in: A.F. Healy and R.W. Proctor (Eds.), Experimental Psychology (pp. 147-176), vol. 4. in: I.B. Weiner (Editor-in-Chief), Handbook of Psychology, Wiley, New York, 2002.

[21] R.L. Klatzky, S.J. Lederman, V. Metzger, Identifying objects by touch: an "expert system", Percept. Psychophys. 37 (1985) 299-302.

[22] R.L. Klatzky, S.J. Lederman, C. Reed, There's more to touch than meets the eye: the salience of object attributes for haptics with and without vision, J. Exp. Psychol. Gen. 116 (1987) 356-369.

[23] R.L. Klatzky, S.J. Lederman, D.E. Matula, Imagined haptic exploration in judgments of object properties, J. Exper. Psychol., Learn., Mem., Cogn. 17 (1991) 314.
[24] R.L. Klatzky, S.J. Lederman, D.E. Matula, Haptic exploration in the presence of vision, J. Exp. Psychol. Hum. Percept. Perform. 19 (1993) $726-743$.

[25] S.M. Kosslyn, W.L. Thompson, When is early visual cortex activated during visual mental imagery? Psychol. Bull. 129 (2003) 723-746.

[26] N.A. Lazar, W.F. Eddy, C.R. Genovese, J.S. Welling, Statistical issues in fMRI for brain imaging, Int. Stat. Rev. 69 (2001) 105-127.

[27] S.J. Lederman, S.G. Abbott, Texture perception: studies of intersensory organization using a discrepancy paradigm and visual versus tactual psychophysics, J. Exp. Psychol. Hum. Percept. Perform. 7 (4) (1981) 902-915.

[28] S.J. Lederman, R.L. Klatzky, Hand movements: a window into haptic object recognition, Cogn. Psychol. 19 (1987) 342-368.

[29] S.J. Lederman, R.L. Klatzky, Relative availability of surface and object properties during early haptic processing, J. Exp. Psychol. Hum. Percept. Perform. 23 (1997) 1680-1707.

[30] S.J. Lederman, C. Summers, R.L. Klatzky, Cognitive salience of haptic object properties: role of modality-encoding bias, Perception 25 (8) (1996) 983-998.

[31] M. Mishkin, Analogous neural models for tactual and visual learning, Neuropsychologia 17 (1979) 139-150.

[32] S. Mutha, H. Chertkow, M. Beauregard, A. Evans, The neural substrate of picture naming, J. Cogn. Neurosci. 11 (1999) 399-423.

[33] S.D. Newman, P.A. Carpenter, S. Varma, M.A. Just, Frontal and parietal participation in problem solving in the Tower of London: fMRI and computational modeling of planning and high-level perception, Neuropsychologia 41 (2003) 1668-1682.

[34] A. Nieto-Castanon, S.S. Ghosh, J.A. Tourville, F.H. Guenther, Region of interest based analysis of functional imaging data, NeuroImage 19 (2003) 1303-1316.

[35] H.L. Pick Jr., Visual coding of nonspatial information, in: R.B. MacLeod, H.L. Pick Jr. (Eds.), Perception: Essays in honor of James J. Gibson, Cornell Univ. Press, Ithaca, NY, 1974.

[36] S.C. Prather, K. Sathian, Mental rotation of tactile stimuli, Cogn. Brain Res. 14 (2002) 91-98.

[37] J. Rademacher, A.M. Galaburda, D.N. Kennedy, P.A. Flilipek, V.S Caviness, Human cerebral cortex: localization, parcellation, and morphometry with magnetic resonance imaging, J. Cogn. Neurosci. 4 (1992) 352.

[38] C.L. Reed, S. Shoham, E. Halgren, Neural substrates of tactile object recognition: an fMRI study, Hum. Brain Mapp. 21 (2004) 236-246.

[39] P.E. Roland, B. O’Sullivan, R. Kawashima, Shape and roughness activates different somatosensory areas in the human brain, Proc. Natl. Acad. Sci. U. S. A. 95 (1998) 3295-3300.

[40] K. Sathian, A. Zangaladze, Feeling with the mind's eye: contribution of visual cortex to tactile perception, Behav. Brain Res. 135 (2002) $127-132$.

[41] P. Servos, S.J. Lederman, D. Wilson, J. Gati, fMRI-derived cortical maps for haptic shape, roughness and hardness, Brain Res., Cogn. Brain Res. 27 (2001) 307-313.

[42] M.A. Srinivasin, R.H. LaMotte, Tactual discrimination of softness, J. Neurophysiol. 73 (1995) 88-101.

[43] R. Vandenberghe, C. Price, R. Wise, O. Josephs, R.S.J. Frackowiak, Functional anatomy of a common semantic system for words and pictures, Nature 383 (1996) 254-256.

[44] M.E. Wheeler, S.E. Petersen, R.L. Buckner, Memory's echo: vivid remembering reactivates sensory-specific cortex, Proc. Natl. Acad. Sci. 97 (2000) 11125-11129.

[45] J. Xiong, S. Rao, J.-H. Gao, M. Waldorff, P.T. Fox, Evaluation of hemispheric dominance for language using functional MRI: a comparison with positron emission tomography, Hum. Brain Mapp. 6 (1998) 42-58.

[46] A. Zangaladze, C.M. Epstein, S.T. Grafton, K. Sathian, Involvement of visual cortex in tactile discrimination of orientation, Nature 401 (1999) 587-590. 